\title{
Missing pearls: Mystery or myth -Case report
}

\author{
Gargi Sengupta ${ }^{1, *}$, Prasanna Kumar Rao², Raghavendra Kini ${ }^{3}$, Gowri P. Bhandarkar ${ }^{4}$, Roopashri Rajesh Kashyap 5 \\ ${ }^{1}$ Post Graduate, ${ }^{2}$ Professor, ${ }^{3}$ Professor and HOD, ${ }^{4,5}$ Reader, Dept. of Oral Medicine \& Radiology, AJ Institute of Dental Sciences, \\ Mangalore, Karnataka, India
}

*Corresponding Author: Gargi Sengupta

Email: gargisengupta2010@gmail.com

\begin{abstract}
Tooth agenesis or failure in development of tooth is one of the most common congenital anomalies. Although absence of one or more teeth is common but absence of multiple teeth is rare. Oligodontia [OD] is one of the rarest genetic disorder or any developmental anomaly which represents the congenital agenesis of either six or more teeth in deciduous and their permanent successors or both dentitions excluding third molars. One of the etiology of congenital absence of teeth which is taken under consideration is rooted in heredity or developmental anomalies. Certain problems are encountered along with OD such as aesthetic, functional, and psychological problems, specifically if the anterior region is involved. OD can exhibit either as a solitary presentation or as a part of a syndrome. Here we present a case of OD in nine year old boy with a complaint of missing mandibular anterior teeth.
\end{abstract}

Keywords: Mandibular, Deciduous, Oligodontia.

\section{Introduction}

One of the most recurrent anomalies of tooth development which is considered -agenesis of either of one or more number of teeth. Although any tooth may be congenitally missing, there is a tendency for certain teeth to be missing more frequently than others. Commonly missing ones: among permanent teeth - third molars (9-37\%), mandibular second premolars $(<3 \%)$, maxillary lateral incisors $(<2 \%)$ and maxillary second premolars and lastly mandibular incisors $(<1 \%) .{ }^{1}$ The prevalence of OD has been reported as $0.3-1 \%$ for anteriors. Incidence of OD usually ranges from $0.08 \%$ to $0.16 \%$. It's a rare condition and their manifestation is common in girls in ratio of 3:2

Down syndrome, Reiger syndrome, Ectodermal dysplasia, Van Der Woude syndrome are one of the syndromes which are most commonly associated with OD. Associated features include conical shaped teeth, microdontia, delayed eruption of permanent teeth, and retained primary teeth. ${ }^{2}$ Usually abnormalities in the skin, nails, eyes, ears or skeleton are noticed when it's a part of a syndrome. ${ }^{4}$

\section{Case Report}

A 9-year- old boy along with his parents reported to the Department of Oral Medicine and Radiology, with the chief complaint of missing upper and lower front teeth since infancy. This condition had been noticed by his parents since early life but did not consult any dentist for the same, they waited for particular age for eruption of permanent teeth. Past medical and dental histories of the patient were noncontributory. No history of noncosanguineous marriage of his parents. When questioned about similar presentation in the family, it was found that his maternal grandfather had same history of congenitally missing mandibular anterior teeth and mother had congenitally missing maxillary lateral incisors bilaterally and delivery of the patient was normal. Extraorally: presence of facial symmetry, straight profile with mild decrease in lower facial height. (Fig. 1A) Fullness of lower lip and slightly protruded relative to the upper lip at closure. Hyperactivity of mentalis muscle upon lip closure which was visible in the skin overlying the chin and presence of deep mentolabial sulcus.

On intra-oral examination revealed that missing of six permanent teeth were not due to extraction, trauma or premature exfoliation of primary teeth rather it was absent since childhood. Maxillary lateral incisors and mandibular incisors (central and lateral) both bilaterally were missing teeth. Third molars were clinically absent. Presence of tongue tie. The maxillary deciduous second molars, mandibular left deciduous second molar were present in dental arches. On palpation: alveolar ridge in the mandibular anterior region was smooth without any eruption protuberance, suggestive of absence of mandibular anterior teeth (Fig. $1 \mathrm{~B}$ and C).

Based on clinical presentation diagnosis of: non syndromic oligodontia was given. Van der Woude syndrome, Ectodermal Dysplasia and Rieger syndrome were considered under differential diagnosis. An orthopantanograph showed no evidence of development of mandibular incisors (central and lateral) bilaterally, maxillary bilateral lateral incisors, mandibular right canine and first premolar. So change of number in relation to missing teeth after radiograph which increased from six to eight congenitally missing teeth. The mandibular deciduous canine were of marked conical shape and there was decrease in dimension of crown mesiodistally. (Fig. 2)

All these differential diagnosis was ruled out because of absence of following features in which OD is associated with common syndromes. 


\begin{tabular}{|l|c|}
\hline \multicolumn{1}{|c|}{ Characteristics } & Syndrome \\
\hline $\begin{array}{l}\text { Hairs were not thin and sparse, } \\
\text { nails were not brittle and no } \\
\text { hindrance in perspiration }\end{array}$ & $\begin{array}{c}\text { Ectodemal } \\
\text { dysplsia }\end{array}$ \\
\hline $\begin{array}{l}\text { Occular examination, no signs of } \\
\text { glaucoma }\end{array}$ & Rieger syndrome \\
\hline $\begin{array}{l}\text { Evidence of cleft lip or cleft palte } \\
\text { or combination of both which } \\
\text { ruled out }\end{array}$ & $\begin{array}{c}\text { Vander Woude } \\
\text { syndrome. }\end{array}$ \\
\hline
\end{tabular}

Patient's maternal grandfather showed oligodontia and mother showed absence of maxillary and mandibular permanent lateral incisors suggestive of hereditary basis to the present defect. Patient is the second child of two from a nonconsanguineous marriage (Fig. 3) Pedigree chart: Patient (III: 2) is second child from non-consanguineous marriage (II:3-mother). Presence of OD in previous two generations from maternal side in one of (Case I: 1) and (Case II: 3) suggest autosomal-recessive-linked inheritance. Finally on basis of both clinical and radiographic presentation: non syndromic oligodontia as final diagnosis was established. Treatment plan considered of: oral prophylaxis, reshaping of conical bilateral mandibular canine, application of pit and fissure sealants, followed by fixed prosthetic replacement of the congenitally missing maxillary and mandibular teeth for esthetic and functional rehabilitation of the patient.

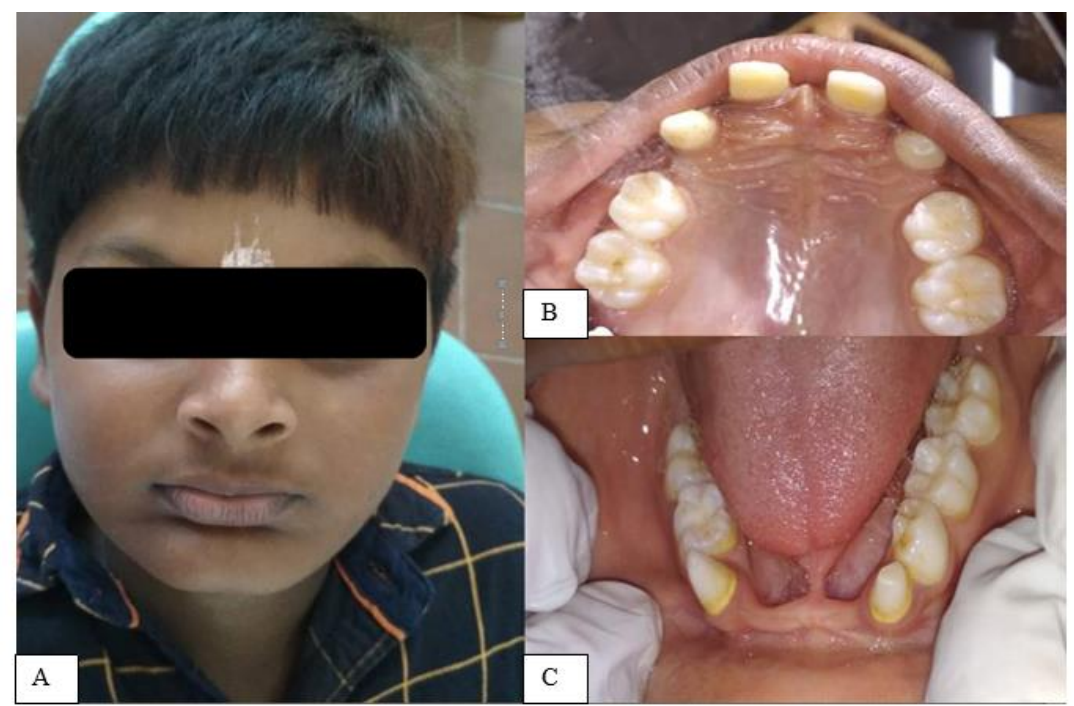

Fig. 1: A straight facial profile; B: Missing permanent lateral incisors, C: OD of mandibular anterior teeth

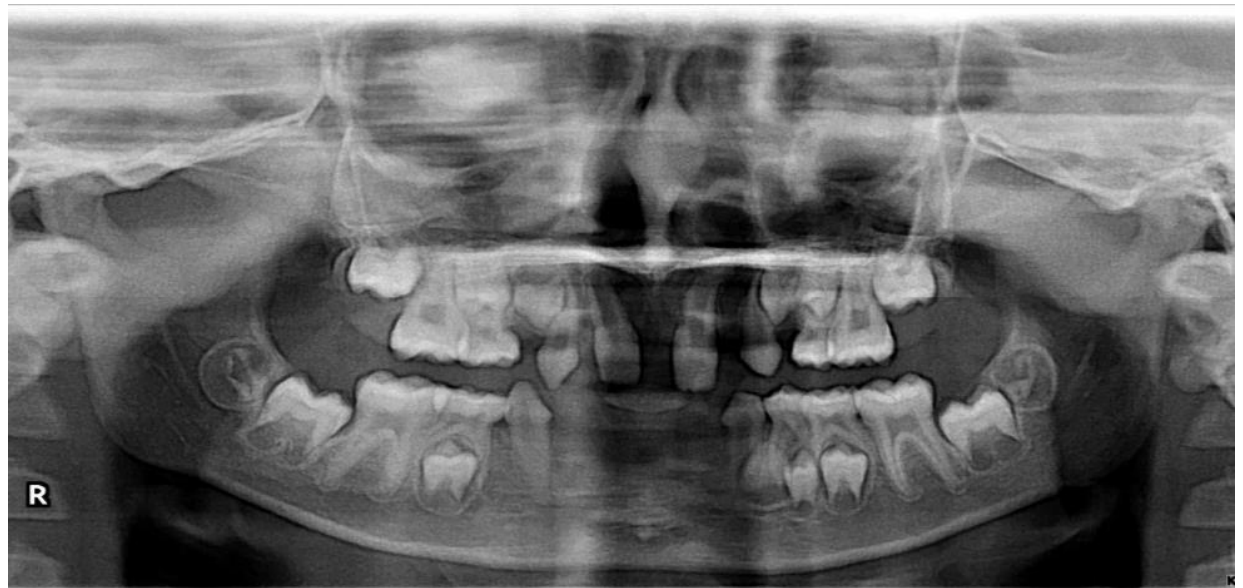

Fig. 2: Panoramic radiograph showing pattern of oligodontia: missing all four permanent mandibular incisors and maxillary lateral incisors; retained primary mandibular canine is seen 


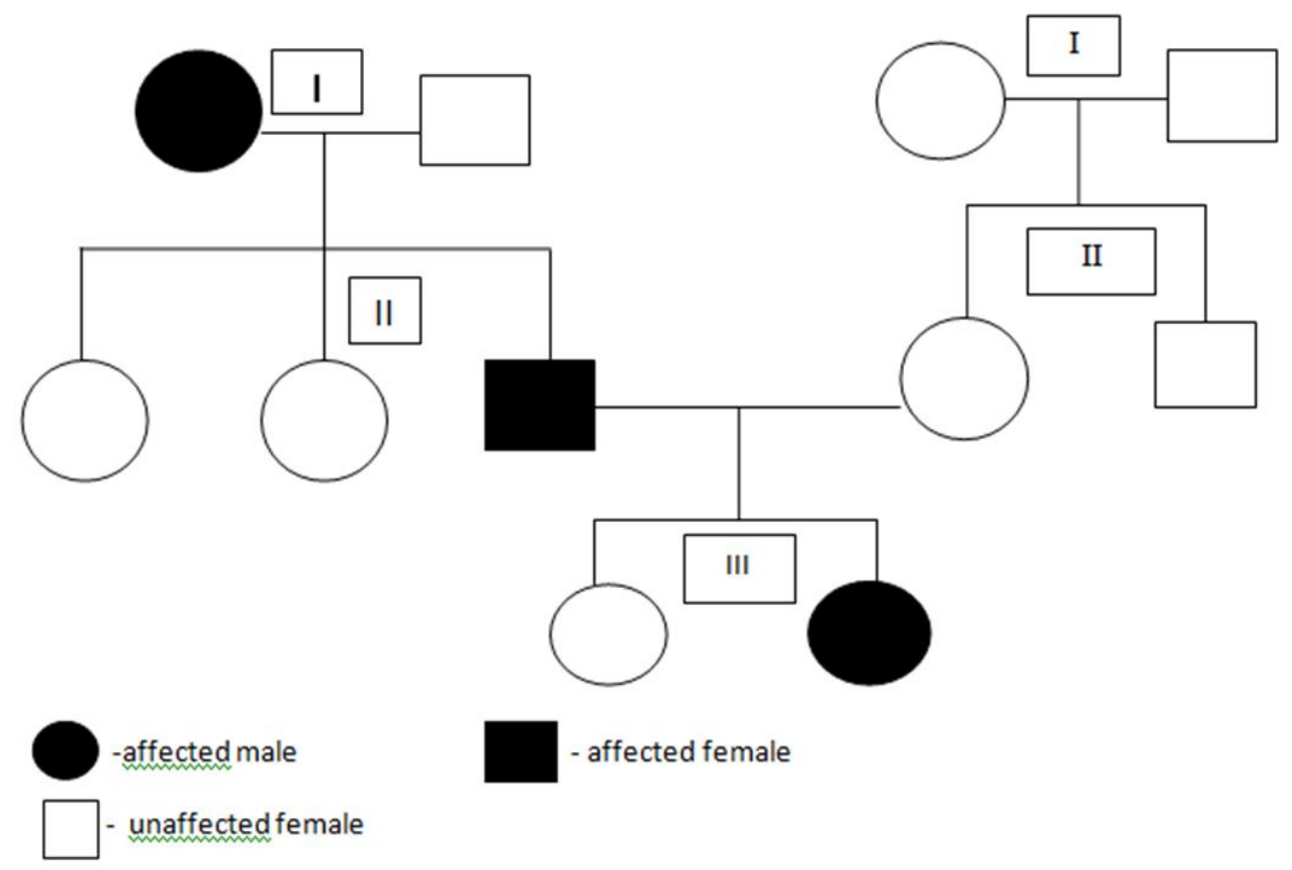

Fig. 3: Case pedigree

\section{Discussion}

One of most common developmental dental anomaly is agenesis of tooth and it might be difficult to manage clinically. A tooth can be considered to be congenitally missing if it cannot be identified clinically or radio graphically and no previous history exists of its extraction due to trauma or premature exfoliation of deciduous teeth. ${ }^{1}$ Based on number of missing teeth (Table 1)

Table 1: Numbers of missing teeth

\begin{tabular}{|l|l|}
\hline \multicolumn{1}{|c|}{ Terms } & \multicolumn{1}{c|}{ Number of Teeth } \\
\hline Hypodontia & $\begin{array}{l}\text { Congenital absence of deciduous or permanent teeth, although specifically it } \\
\text { describes the absence of one to six teeth excluding third molars }\end{array}$ \\
\hline Oligodontia (OD) & Refers to the absence of more than six teeth, excluding third molars \\
\hline Anodontia & Loss of all teeth. \\
\hline
\end{tabular}

Oligodontia has been classified (Table 2)

Table 2: Classification of oligodontia

\begin{tabular}{|l|l|}
\hline I & $\begin{array}{l}\text { Associated with Syndrome/Nonsyndrome } \\
\text {-Isolated or non-syndromic /familial and } \\
\text { - Syndromic }\end{array}$ \\
\hline II & $\begin{array}{l}\text { Basis on Clinical/ Radiographic Appearance } \\
\text {-True OD - absence of teeth clinically, might be presence of tooth buds. } \\
\text {-False OD - congenital absence of tooth buds visible on radiograph. }\end{array}$ \\
\hline
\end{tabular}

OD is an unusual condition affecting $0.1-0.2 \%$ of population and its manifestation is common in girls in ratio of 3:2. ${ }^{2}$ Both forms of congenital absence of tooth have been connected with mutations of related genes. Non-syndromic or familial hypodontia is more common comparatively than syndromic type. This condition can follow autosomal (dominant, recessive) or X-linked patterns of inheritance, with considerable variation in both penetrance and expressivity. More common cases of incisor-premolar type of hypodontia, genetic analysis has excluded MSX1 gene as one of causative locus for this type of hypodontia ${ }^{3}$
The underlying cause is due to failure in proliferation of tooth buds from dental lamina. Hereditary or environmental are considered to attribute towards this condition.

Hereditary: condition can be transmitted as autosomal dominant, autosomal recessive, or X-linked. In early tooth formation genes which are related MSX-1 and PAX gene; therefore, mutation in any one of the gene can result in agenesis.

Environmental factors such as: trauma, infection (rubella), exposure to drugs such as (thalidomide), hormonal influences, previous irradiation, or presence of tumor are some of the causative factors. ${ }^{5}$ 
In this case report, there were eight congenitally missing teeth. As such no other physical characteristics of a syndrome were present. Missing teeth were bilateral maxillary laterals, four mandibular incisors (central and lateral), canine and premolar, and all third molars. Literature review reveals that most common congenital missing teeth are mandibular second premolars followed by maxillary second premolars and maxillary laterals and among them missing of canine is very rare. ${ }^{5}$ Interestingly, in present case, the mandibular canines were congenitally missing which brings the need for reporting this case and to bring awareness of the same among dentists. There have been strong impact of OD on declining the level of confidence, specially in high school children and at workplace, due to unsatisfied appearance of smile owing to weak performance. Adding up to all this, problems such as mastication, speech, and malocclusion do exist in these kinds of patients, thus deteriorating one's quality of life. Overall, this affects an individual's emotional and psychological balance. ${ }^{5}$

OD condition deals with various disturbances like malocclusion, altered facial presentation which may cause psychological desolation and might have difficulty in deglutition and in conversation which shouldn't be neglected. Multidisciplinary approach is appreciated depending on intensity of hypodontia (oligodontia or anodontia). Early diagnosis, comprehensive planning and timing of each phase of individual treatment plays very important role for successful outcome in such conditions. To rule out any syndrome certain investigations has to be carried out such as complete clinical history including medical and radiological should be documented in case of tooth agenesis. ${ }^{2}$

\section{Conclusion}

OD is mostly considered to be associated with several syndromes but non syndromic aspect of it should also be considered. Multidisciplinary approach helps to have successful outcome in treating and managing of patient with OD. Age of the patient plays a pivotial role in management. A patient presenting with missing teeth suffers not only from deglution, phonetics and appearance but also psychological distress, as it can lower the self-esteem of an otherwise healthy individual. Dental fraternity should aim at treating the condition as early as possible and achieve both prosthetic and aesthetic functionality of teeth and at the appropriate time jointly by interdisciplinary approach to improve oral health and psyche of the child. Early systematic intervention will help in improving esthetics, speech, mastication, and social life.

\section{Conflict of Interest: Nil.}

\section{References}

1. Pannu P, Galhotra V, Ahluwalai P, Gambhir RS. NonSyndromic Oligodontia in Permanent Dentition : A Case Report. 2014;48(3):3-6.

2. Tangade P, Batra M. Case Report Non Syndromic Oligodontia : Case Report. :219-21.

3. Shimizu T, Maeda T. Prevalence and genetic basis of tooth agenesis. Jpn Dent Sci Rev. 2009;45(1):52-8.

4. Bural C. Multidisciplinary treatment of non- syndromic oligodontia. 218-26.

5. Donald PM, Arora A, George R. Nonsyndromic Form of Oligodontia in a Chinese Male Patient : A Rare Case Report. 2017;2017-9.

How to cite this article: Sengupta G, Rao P. K, Kini R, Bhandarkar G. P, Kashyap R. R. Missing pearls: Mystery or myth - Case report. Int J Maxillofac Imaging. 2018;4(4):140-143. 